Military Technical College Kobry El-Kobbah, Cairo, Egypt

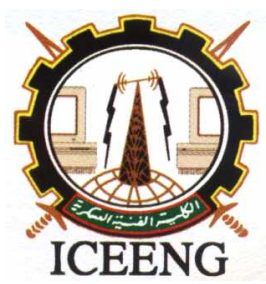

\author{
$6^{\text {th }}$ International Conference \\ on Electrical Engineering \\ ICEENG 2008
}

\title{
A Novel Approach for the Assessment of the Reliability of Distributed Generation Systems
}

\author{
By \\ El-Said El-Bagoury \\ Y. G. Hegazy \\ M. A. Mostafa \\ M. A. Badr
}

\section{Abstract:}

This paper presents a novel algorithm for adequacy assessment of electrical distribution system including distributed generation. A comparison between the proposed technique and the state duration sampling technique is performed. The proposed technique is integrated with Monte Carlo simulation method to carry out reliability analysis of typical distribution system. The results obtained are presented and discussed.

\section{Keywords:}

Distributed generation, Monte Carlo simulation, and Reliability

* Department of Electrical Power and Machines, Faculty of Engineering, AinShams University, Cairo, Egypt 


\section{INTRODUCTION}

At present the electric power industry is undergoing considerable charge with respect to structure, operation, and regulation [1-2]. The various electric utility acts introduced in different countries have initiated the restructuring process and the traditional vertically integrated utility structure consisting of generation, transmission, and distribution functions has been dismantled [3]. Due to the wide spread use of distributed generation (DG) which is defined as small scale power generation located at or near to the load being served, several system operating issues have come into sight. These concerns involve both the benefits of using $\mathrm{DG}_{\mathrm{s}}$ and the problem associated with the wide implementation of DG units in a well established system for several decades. The concept of implementing small generation in the distribution system has been in practice for several years as backup, standby and standalone generation. However, as a result of the new modern technology achieved in the generation industry and the benefits expected from this technology implementation, a wide spread use of these DG units operated in the system has become a fact [4]. The main reasons behind the expected widespread of DG are:[5,6]

- The deregulation in the power market, which encourage public investment to sustain the

- Development in the power demand. This development has led to the breaking up of investments

- The emergence of new generation techniques with small rating ,ecological benefits, increased profitability ,and which can be combined with heat generation.

- The saturation of the existing networks and the continuous growth of the demand.

The difficulties in the operation and distribution systems are still the main challenges associated with parallel operation of DG with these systems [7]. In the real life systems , the operation of these DG units undergoes different scenarios according to the strategies of the electricity producers, the needs of the consumers and the zonal load time variation characteristics. Therefore, some uncertainties are introduced in the operation of such units and thus, stochastic modeling of systems involving DG units becomes of a great interest[8] .

The sources of uncertainty in the operation of DG connected systems at a certain hour of the day include the number of the running DG units at this hour, the locations of these units and the power exported to the system by these units. These uncertainties affect the modeling and evaluation of the system capacity, generation scheduling, and reliability adequacy assessment .

In this paper the proposed technique incorporated Monte Carlo simulation and give a comparison with the State Sampling technique. The adequacy assessment of the system and the calculation of reliability indices are performed using the proposed 
technique ,also this study examine the impact of DG units with different capacity and location on the excepted reliability indices.

Billinton and Wang in [9] calculate the reliability indices: average load failure rate , average load outage rate, and average load outage time, also determine SAIFI, CAIDI, ASAI, and SAIDI of distribution system of the RBTS using sequential Monte Carlo simulation .They performed analysis with both analytical and simulation technique where the simulation approach can provide information on the load point and system indices that the analytical techniques can not provide.

Chowdhury and el. in [3] determine the size of distributed generator or combination of smaller distributed units by adding to the existing system and compare its effect on the reliability of the system with a adding extra feeder from substation to the existing system. They verify the presence of DG is enhancing the system reliability, where the duration of interruptions per customer per year and the duration of system interruptions per year are reduced.

Hegazy and Salama in [4] General procedure to assess the ability of the system power capacity to meet the total demand is presented and implemented in atypical case study where several distributed generation units are running in parallel within a system and the system margins and the average amount of unsupplied loads are estimated using Monte Carlo simulation. The results obtained showed that DG can enhance the overall capacity of the distribution system and be used an alternative to substation expansion in case expected demand growth.

Kaigui and el in[10] they presents algorithm, which can be used to evaluate the reliability of complex medium voltage, an approach to searching for the shortest paths from a node to source and between any two nodes is presented ,based on graph theory and structural features. The approach can be used to consider the effect of breakers ,fuses ,disconnect switches ,alternative supplies and replacing transformers in addition to other system

\section{The Proposed ApProACH}

The distribution system under study is comprised of two centralized substation the ratings are $6 * 100$ and $4 * 150 \mathrm{MW}$, so that the total generated power is $1200 \mathrm{MVA}$. The data required to construct the load model are presented as a part of IEEE reliability test system in[11,12] with the peak system load is $1000 \mathrm{MW}$. Let $\mathrm{P}_{\mathrm{T}}$ is the total power received from substation transformers, and $\mathrm{P}_{\text {tr }}$ is the output power of each transformer ,where

$$
\mathrm{P}_{\mathrm{T}}=\sum \mathrm{P}_{\mathrm{tr}}
$$


$P_{\text {tr }}$ is vary randomly in the range from 90 to $100 \%$ of its nominal capacity (100 or 150 MW) .Adding DG (distributed generator) units to operate in parallel with the substation transformers will increase $\mathrm{P}_{\mathrm{S}}$ (distribution system power capacity) where

$$
\mathrm{P}_{\mathrm{S}}=\mathrm{P}_{\mathrm{T}}+\mathrm{P}_{\mathrm{DG}}
$$

$P_{D G}$ is the total power received from DG units.

$$
\mathrm{P}_{\mathrm{DG}}=\sum \mathrm{P}_{\mathrm{Gi}}
$$

The system capacity curve is superimposed on the chronological load curve in $[9,10]$ to obtain the system available margin model, then the reliability indices can be calculated using Monte Carlo method .In each sample year( $i)$, the loss of load duration (LLD $\left.{ }_{i}\right)$ in $\mathrm{hr}$, the loss of load occurrence $\left(\mathrm{LLO}_{\mathrm{i}}\right)$, and the energy not supplied $\left(\mathrm{ENS}_{\mathrm{i}}\right)$ in $\mathrm{MWh}$. The reliability indices in $\mathrm{N}$ sampling years can be estimated using the following equations:

(1) Loss of Load Expectation (LOLE), hr/yr

$$
\text { LOLE }=\sum_{i=1}^{N} \frac{L L D i}{N}
$$

(2) Loss of Energy Expectation (LOEE), MWh/yr

$$
\mathrm{LOEE}=\sum_{i=1}^{N} \frac{E N S i}{N}
$$

(3) Loss of load Frequency (LOLF) occ./yr

$$
\mathrm{LOLF}=\sum_{i=1}^{N} \frac{L L O i}{N}
$$

In this paper, a random-number generator is employed to generate an integer $\left(\mathrm{k}_{1}\right)$, where $\mathrm{k}_{1} \mathrm{C}[1, \ldots, 10]$ to represent the expected number of transformers in their "on state." and Transformers' rating are then identified, by generating a random sequence of integers $\left(\mathrm{k}_{1}\right)$, where this sequence represents the transformer number and $\mathrm{P}_{\mathrm{T}}$ is calculated ,the sequence above is repeated each hour in year.

Also another random-number generator is employed to generate an integer $\left(\mathrm{k}_{2}\right)$, where $\mathrm{k}_{2} \mathrm{C}[1, \ldots, \mathrm{M}]$ to represent the expected number of DGs in their" on state." where $\mathrm{M}$ is the total no of DGs , and DGs ' rating are then identified, by generating a random sequence of integers $\left(\mathrm{k}_{2}\right)$, where this sequence represents the $\mathrm{DG}$ number and $\mathrm{P}_{\mathrm{DG}}$ is calculated . 
In the proposed algorithm, the power exported from each DG unit to the distribution system is assumed to be varying randomly. To simulate this feature, the magnitude of the generator power is calculated each time the simulation is recalled. The DG nominal rating is multiplied by a random number, where

is a uniformly distributed random number $\mathrm{U}$, where $\mathrm{U}$ is uniformly random number sequence generally between $[0,1]$. However, the minimum value of $U$ can be adjusted according to the historical primary distribution system where

$$
\mathrm{P}_{\mathrm{Gi}}=\mathrm{P}_{\mathrm{Ri}} * \mathrm{U}
$$

$\mathrm{P}_{\mathrm{Ri}}$ is rated power of each $\mathrm{DG}$, and $\mathrm{P}_{\mathrm{Gi}}$ is generated power of $\mathrm{DG}$ at each hour in the year.MATLAB programs is used and m-files are performed by author for this study.

The flow chart of the Monte Carlo base algorithm is showing in fig. 1 and step by step procedure as follows:

1) Model the load curve, rating of substation transformer, and the max. no. of DGs (M).

2) Estimate the hourly operating transformer number, sequence, and power and run the model:

3) Generate random integer $k 1$ each hour in the year ,to represent the expected no. of transformer operating .

4) Generate random sequence of the selected integer k1 each hour in the year to represent any transformer $\mathrm{ON}$.

5) Generate random power multiplier Utr to calculate each transformer export power, the min value of Utr is 0.9 .

6) Calculate PT

a) Estimate the hourly operating DG number, sequence, and power and run the model:

b) Generate random integer k2 each hour in the year ,to represent the expected no. of DG operating .

7) Generate random sequence of the selected integer $k 2$ each hour in the year to represent any transformer $\mathrm{ON}$.

8) Generate random power multiplier $U_{D G}$ to calculate each DG export power, the min value of $U_{D G}$ is adjusted.

9) Calculate $P_{D G}$

a) Calculate PS , the margin, and the reliability indices(LOLE,LOEE,LOLF).

b) Perform the Monte Carlo convergence till all indices reach saturation.

c) Repeat step-2 for all hours of the year. 


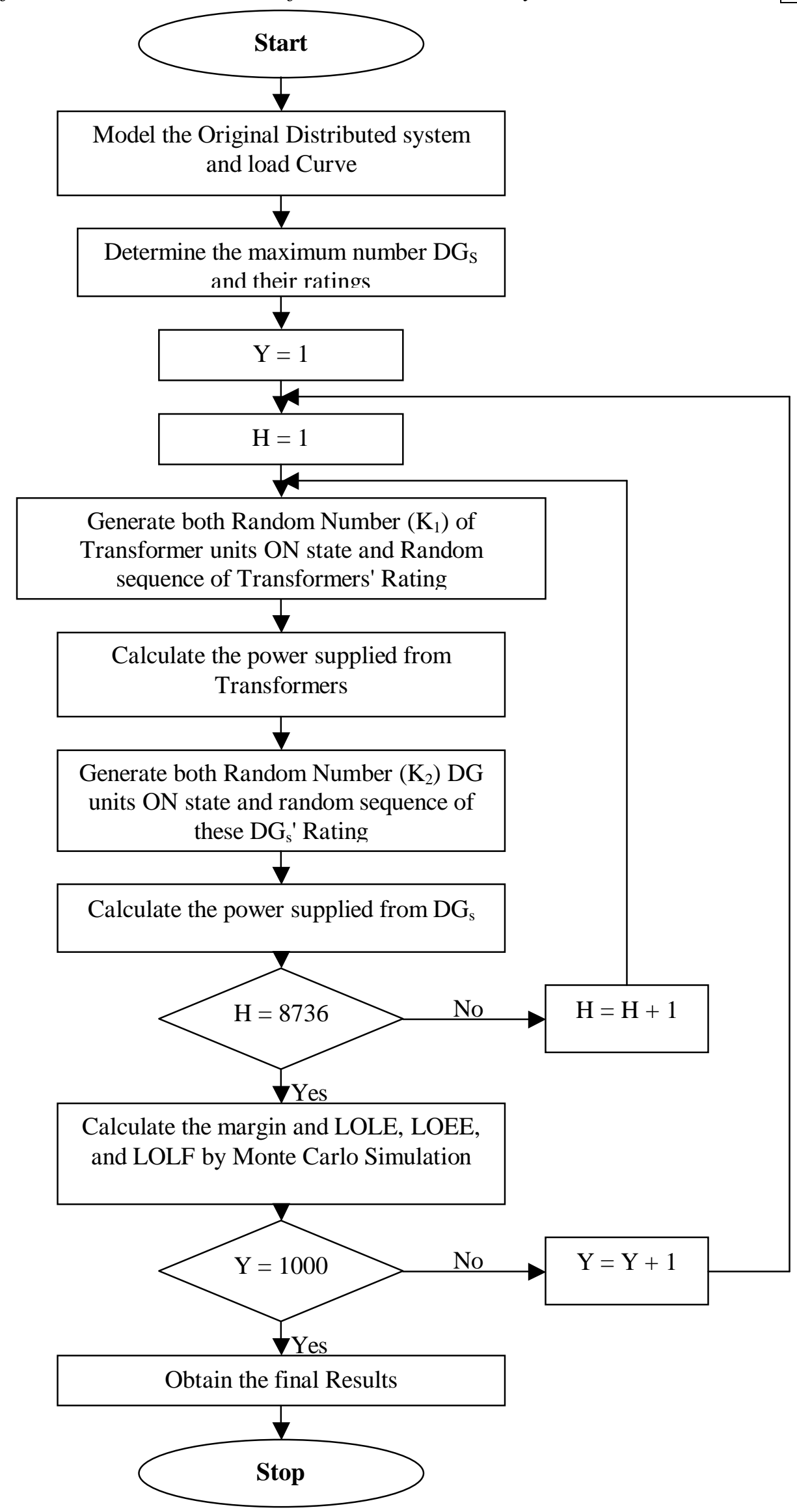




\section{Validation of The Proposed Method}

The IEEE RTS has 32 generating units ranging from $12 \mathrm{MW}$ to $400 \mathrm{MW}$ as in table 1.the annual load curve of 8736 hourly load points with system peak load $2850 \mathrm{MW}$, the results of the three reliability indices (LOLE,LOEE,LOLF) are calculated in [12] using State Duration Sampling Method and the results shown in fig.2 In the proposed method divide the generators into two groups :

The first is the largest units from 155 to $400 \mathrm{MW}$ and the number of their 10 units. These units operates as base units like substation transformer, where the minimum number of units $\mathrm{ON}$ is 9 units.

The second group is units from 12 to $100 \mathrm{MW}$ and the number of their 22 units. These units operates as a peak units like DGs, where the minimum number of units ON is 15 units $(68 \%)$. The proposed algorithm is applied and the reliability indices shown in fig.3 , the comparison between the results of the two methods in table 2 .

Table 1 Generating unit data

\begin{tabular}{|c|c|c|}
\hline No. & $\begin{array}{c}\text { Unit } \\
\text { Size } \\
\text { MW }\end{array}$ & $\begin{array}{c}\text { Number } \\
\text { of units }\end{array}$ \\
\hline 1 & 12 & 5 \\
\hline 2 & 20 & 4 \\
\hline 3 & 50 & 6 \\
\hline 4 & 76 & 4 \\
\hline 5 & 100 & 3 \\
\hline 6 & 155 & 4 \\
\hline 7 & 197 & 3 \\
\hline 8 & 350 & 1 \\
\hline 9 & 400 & 2 \\
\hline Total & 3405 & 32 \\
\hline
\end{tabular}



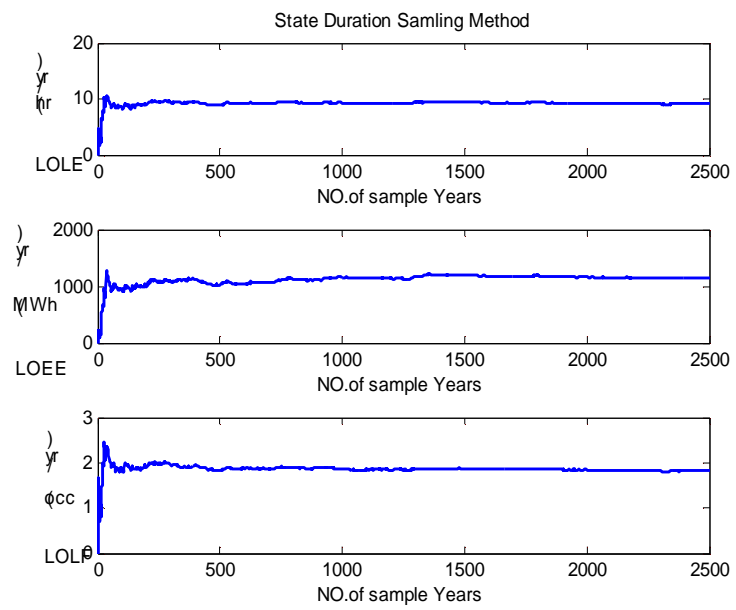

Fig.2 LOLE,LOEE,LOLF vs. the number of sample years
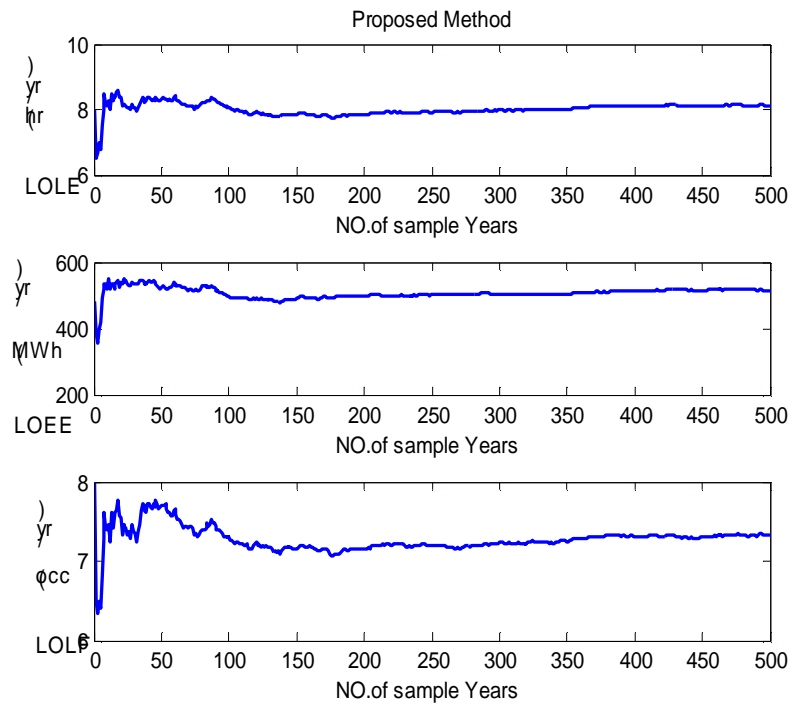

Fig.3: LOLE, LOEE and LOLF vs. the number of sample years

Table 2 comparison between the two methods

\begin{tabular}{|l|c|c|}
\hline \multicolumn{1}{|c|}{ Index } & $\begin{array}{c}\text { State } \\
\text { Duration } \\
\text { Method }\end{array}$ & $\begin{array}{c}\text { Novel } \\
\text { Method }\end{array}$ \\
\hline LOLE (hr/yr) & 9.2341 & 8.1260 \\
LOEE & 1134 & 515 \\
(MWh/yr) & 1.92 & 7.3320 \\
LOLF (occ./yr) & & \\
\hline
\end{tabular}




\section{CaSe Study ANd Results}

The distribution system consists of two substation 10 transformers with ratings $(6 \times 100$, and $4 \times 150 \mathrm{MW}$ ), the maximum capacity is $1200 \mathrm{MW}$, where the minimum number of transformer (on state)is (8 from 10). The maximum load is $1000 \mathrm{MW}$

The results of calculation of LOLE,LOEE ,LOLF without adding DGs shown in fig.4 , the value of LOLE equal 21.18 (hr/yr), LOEE equal 498.73 (MWh/yr), and LOLF equal 15.8 (occ./yr).

Adding one DG with different ratings $2 \%$ of the maximum load capacity (20 $\mathrm{MW}), 4 \%(40 \mathrm{MW}), 7 \%(70 \mathrm{MW})$, and 10\% (MW) to the generated power from transformer substation will enhance the reliability indices.

Fig.5 show LOLE with adding one DG 2\%,4\%,7\%, and $10 \%$ where the generated power from DG is full capacity (UDG $=1.0$ ), from this figure it can be observed that the value of LOLE decreased from 21.18(hr/yr)in case of without DG to 10.7 (hr/yr), with adding DG $10 \%$. The same tendency of decrease of LOLE is observed in fig. 6 , where $\mathrm{UDG}=0.7(\mathrm{PGi}=\mathrm{PRi} * \mathrm{UDG})$, the comparison of with and without Adding one DG on the value of LOLE is shown in table 3.
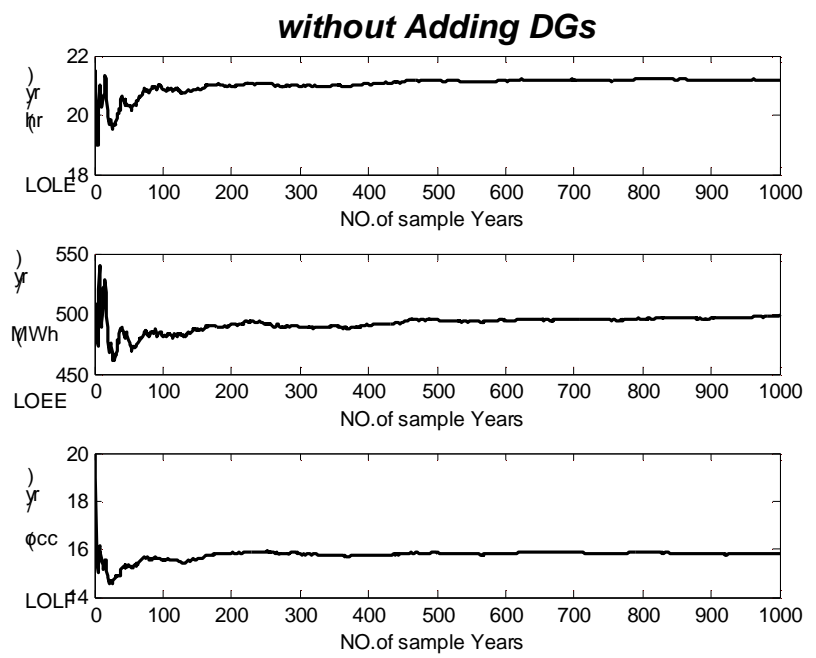

Fig.4 LOLE,LOEE,LOLF vs. the number of sample years, with min 8 transformer ON

Table 3 the value of LOLE (hr/yr)

\begin{tabular}{|c|c|c|c|c|c|}
\hline $\begin{array}{c}\text { Without } \\
\text { DG }\end{array}$ & \multicolumn{5}{|c|}{ Adding one DG } \\
\hline \multirow{4}{*}{21.18} & $\mathrm{U}_{\mathrm{DG}}$ & $\mathrm{DG}$ & $\mathrm{DG}$ & $\mathrm{DG}$ & $\mathrm{DG}$ \\
& & $2 \%$ & $4 \%$ & $7 \%$ & $10 \%$ \\
\cline { 2 - 6 } & 1.0 & 15.76 & 12.61 & 11.14 & 10.70 \\
\cline { 2 - 6 } & 0.7 & 16.09 & 14.17 & 12.13 & 10.96 \\
\hline
\end{tabular}




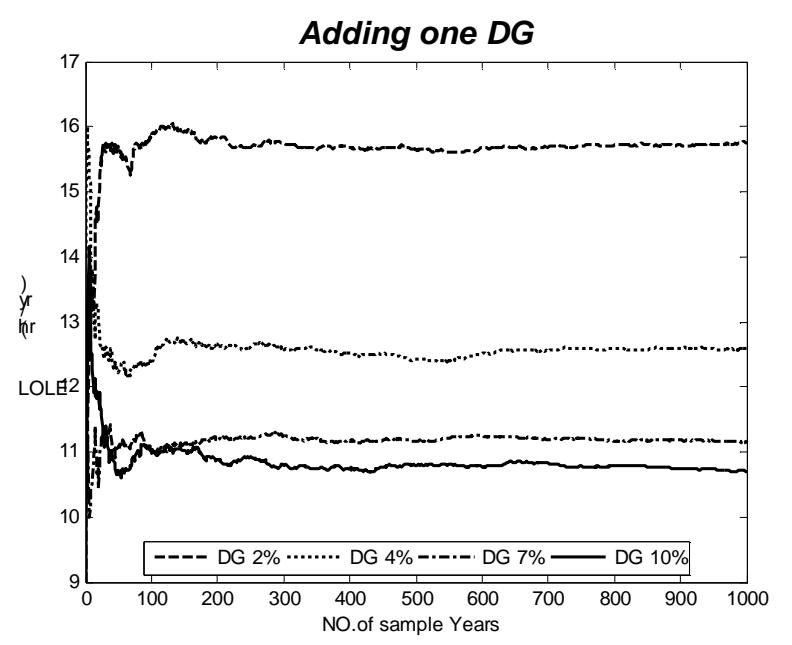

Fig.5 The DG power (100\%) of its capacity

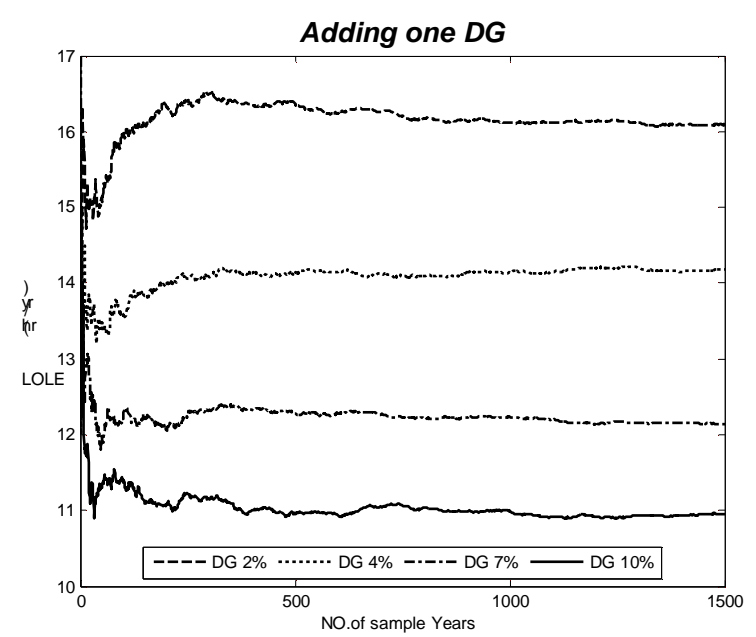

Fig.6 The DG power (70 to100\%) of its capacity

Adding two DGs with ratings capacity $(2 \%, 2 \%),(2 \%, 4 \%)$, and $(4 \%, 4 \%)$ to the capacity of substation transformer have more improvements on the reliability indices than one DG ,these verified in figures $7,8,9,10$.

Fig.7 shows the values of LOLE with different probabilities of the two DGs capacity , where $\mathrm{U}_{\mathrm{DG}}=1.0$, and minimum no DG is ON state at any hour .In other words at each hour the number of DGs in ON state are (zero or one or two).

While fig. 8 describe the minimum one from two DGs is ON at each hour(no. of DGs $\mathrm{ON}$ are one or two), and $\mathrm{U}_{\mathrm{DG}}=1.0$ 


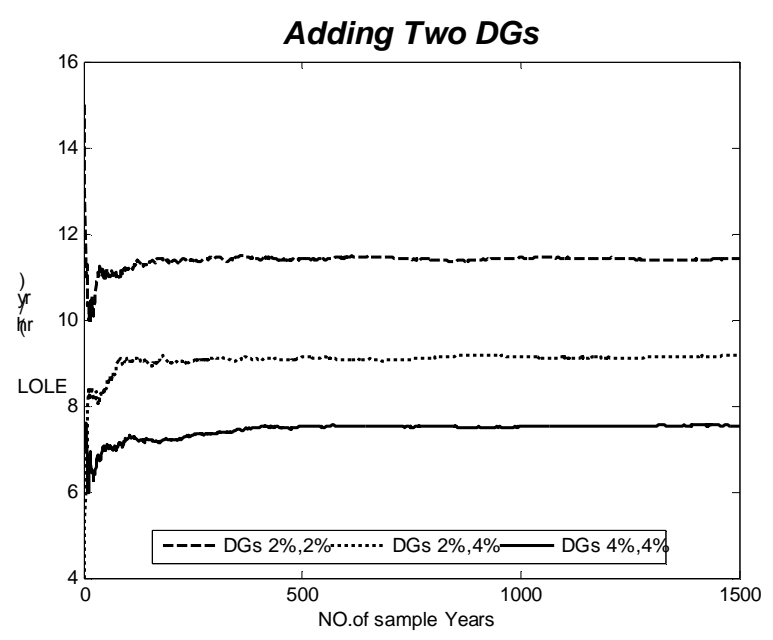

Fig.7 The DGs power (100\% )of their capacity, and min. no DG is ON

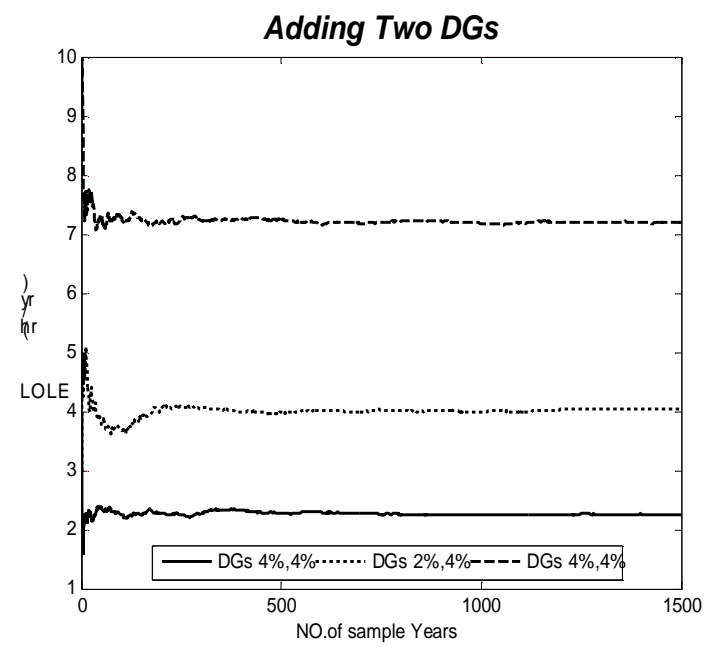

Fig.8 The DGs power (100\%) of their capacity, and min. one DG is ON

Fig.9 shows the effect of adding two DGs and UDG $=0.7$, where the minimum number of DGs in ON state is zero, but fig.10 draws with minimum DGs ON is one . The results of the values of LOLE in figures 7,8,9, and 10 . 


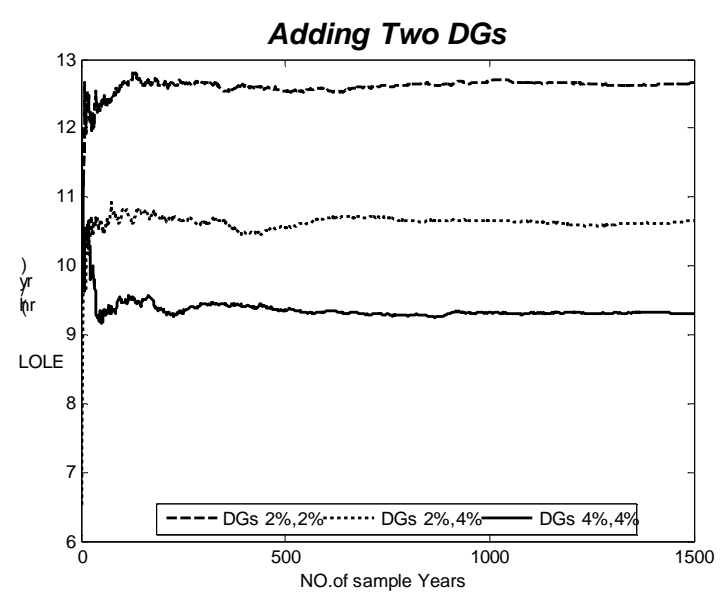

Fig.9 The DGs power (70 to100\%) of their capacity, and min. no DG is ON

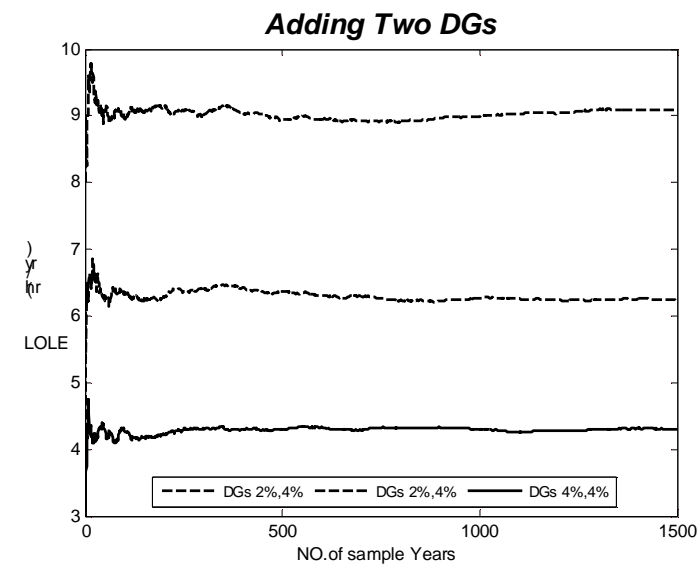

Fig.10 The DGs power (70 to $100 \%$ )of their capacity, and min. one DG is ON

\section{CONCLusions}

This paper presents a novel algorithm that incorporates the modeling reliability aspects of distributed generation systems and substation transformer connected to the distribution systems. The algorithm provides the hourly system': centralized power generation from substation, and total DG penetration levels. Monte Carlo simulation technique is employed in the algorithm to cover all possible operation conditions of the systems. A typical case study is analyzed with three penetration levels of DGs.The first adding one DG with different capacity and different percentage of this capacity.

The second adding two DGs with different probabilities of the number of their On state( 0 or 1 or 2 ). The third case adding three DGs and consider all possibilities of the 
two previous cases with the minimum number in $\mathrm{ON}$ state are $(0$ or 1 or 2 or 3).According with the results obtained by the algorithm, it can be verified DG can enhance the overall capacity of the distribution system and increase its reliability. Finally in our case study the critical penetration level of DGs to improve the system reliability to approximately one hundred percent is two DGs with capacity four percent of the maximum load capacity on all the time.

\section{REFERENCES}

[1] S.V.Vadari,J.D.Hammerly, "New faces and functions in the competitive market" IEEE Comput. Appl.power,vol.10,pp. 47-52,Jan.1997.

[2] R.Billinton,L.salvaderi,J.D.McCally,H.chao,Th.Seitz,R.N.Allan,J.Odom, and C.Fallon, "Reliability issues in today's electric power utility environment" IEEE Trans. Power Syst.,vol.12,pp. 1708-1714,Nov.1997.

[3] A.A.Chowdhury,Sudhir Kumar Agrawal,and Don O.Koval, "Reliability of Distributed generation in Conventional Distribution Systems Planning and Analysis" IEEE Trans. Indus.Appl..,vol.39, No.5,pp. 1493-1497,Oct.2003.

[4] Y.G. Hegazy, M.M.A.Salama,A.Y.Chikhani, "Adequacy Assessment of Distributed Generation Systems Using Monte Carlo Simulation," " IEEE Trans. Power Syst.,vol.18, No.1,pp. 48-52,Feb.2003.

[5]T.Ackemann,G.Andersson, and L.Soder, " Electricity market regulations and their impact on the distributed generation" in Proc.Int.Conf.Elect.Utility Deregulation Restructuring Power Technol., London, U.K.,Apr. 2000 , pp. 608613 .

[6] N.Hadjsaid, J.Canard,F.Dumas, "Dispersed generation increases the complexity of controlling and maintaining the distribution systems," " IEEE Comput. Appl.power,vol.12,pp. 23-28,Apr.1999.

[7]P.P.Barker, "Determining the impact of Distributed Generation on power Systems: Part 1-radial distribution systems," Proceeding of the IEEE PES Summer meeting 2000,pp. 1032-1037.

[8] Walid El-Khatam, Y.G.Hegazy,M.M.A.Salama, "Investing Distributed Generation Systems Performance using Monte Carlo Simulation” IEEE Trans. Power Syst.,vol.12,No.2,pp. 524-532,May.2006.

[9] R.Billinton and P.Wang, "Teaching Distribution System Reliability Using Monte Carlo Simulation"IEEE Trans. on Power System,Vol.14,No.2 May 1998.

[10] Kaigui Xie, Jiaqi Zhou, and R.Billinton, "Reliability evaluation algorithm for complex medium voltage electrical distribution networks based on the shortest path"IEE Proc.Gener.Distrib.,vol. 150, No. 6, pp.686-690,Nov. 2003. 
[11] R.N.Allan,R.Billinton, and N.M.Abdel-Gaead, "The IEEE Reliability Test System Extension to and Evaluation os the generating System," IEEE Trans. on PWR,vol.1,No.4,pp.1-7,1986.

[12] R.Billinton, Wenyuan Li, "Reliability assessment of Electric power systems Using Monte Carlo Methods" Book of Plenum Press, New York and London 1994. 